\title{
Human Mitochondrial DNA Replication
}

\author{
Ian J. Holt and Aurelio Reyes \\ MRC Mitochondrial Biology Unit, Cambridge, United Kingdom \\ Correspondence: ih@mrc-mbu.cam.ac.uk
}

Elucidation of the process of DNA replication in mitochondria is in its infancy. For many years, maintenance of the mitochondrial genome was regarded as greatly simplified compared to the nucleus. Mammalian mitochondria were reported to lack all DNA repair systems, to eschew DNA recombination, and to possess but a single DNA polymerase, polymerase $\gamma$. Pol $\gamma$ was said to replicate mitochondrial DNA exclusively via one mechanism, involving only two priming events and a handful of proteins. In this "strand-displacement model," leading strand DNA synthesis begins at a specific site and advances approximately two-thirds of the way around the molecule before DNA synthesis is initiated on the "lagging" strand. Although the displaced strand was long-held to be coated with protein, RNA has more recently been proposed in its place. Furthermore, mitochondrial DNA molecules with all the features of products of conventional bidirectional replication have been documented, suggesting that the process and regulation of replication in mitochondria is complex, as befits a genome that is a core factor in human health and longevity.

$\mathrm{H}$ uman mitochondrial DNA (mtDNA) typically forms 5 - $\mu \mathrm{m}$ circles, or 16.5 kilobases, 1 genome in length. Outside the animal kingdom, mtDNA varies prodigiously in size and form, yet it invariably exists to provide the cell with essential components of the respiratory chain (and ATP synthase). Hence, mutations of mtDNA can cause respiratory chain dysfunction, which in turn causes rare diseases in plants (Levings and Pring 1976; Newton and Coe 1986) and humans (Holt et al. 1988). Budding yeast has the option of growing anaerobically and so can manage without mtDNA (Goldring et al. 1970), and remarkably vertebrate cells cultivated in the laboratory can achieve the same feat, on a diet of glucose far less concentrated than a typical soda (Desjardins et al. 1985; King and
Attardi 1989). Nevertheless, not even a simple free-living multicellular organism, such as the nematode worm, can survive without mtDNA (Bratic et al. 2009).

The mtDNA of all vertebrates is very similar to that of humans, almost all the noncoding DNA is concentrated in one region, of little more than a kilobase, known as the major noncoding region, or NCR (Fig. 1). Although most of the mtDNA sequence variation among humans is found in the NCR, it is not evenly distributed but concentrated in three hypervariable sections (Ingman et al. 2000). In the early 1970s, two groups reported that many mtDNA molecules contain a triple-stranded region, which forms a displacement or D-loop (Arnberg et al. 1971; Kasamatsu et al. 1971). The D-loop

Editors: Stephen D. Bell, Marcel Méchali, and Melvin L. DePamphilis

Additional Perspectives on DNA Replication available at www.cshperspectives.org

Copyright (C) 2012 Cold Spring Harbor Laboratory Press; all rights reserved; doi: 10.1101/cshperspect.a012971

Cite this article as Cold Spring Harb Perspect Biol 2012;4:a012971 


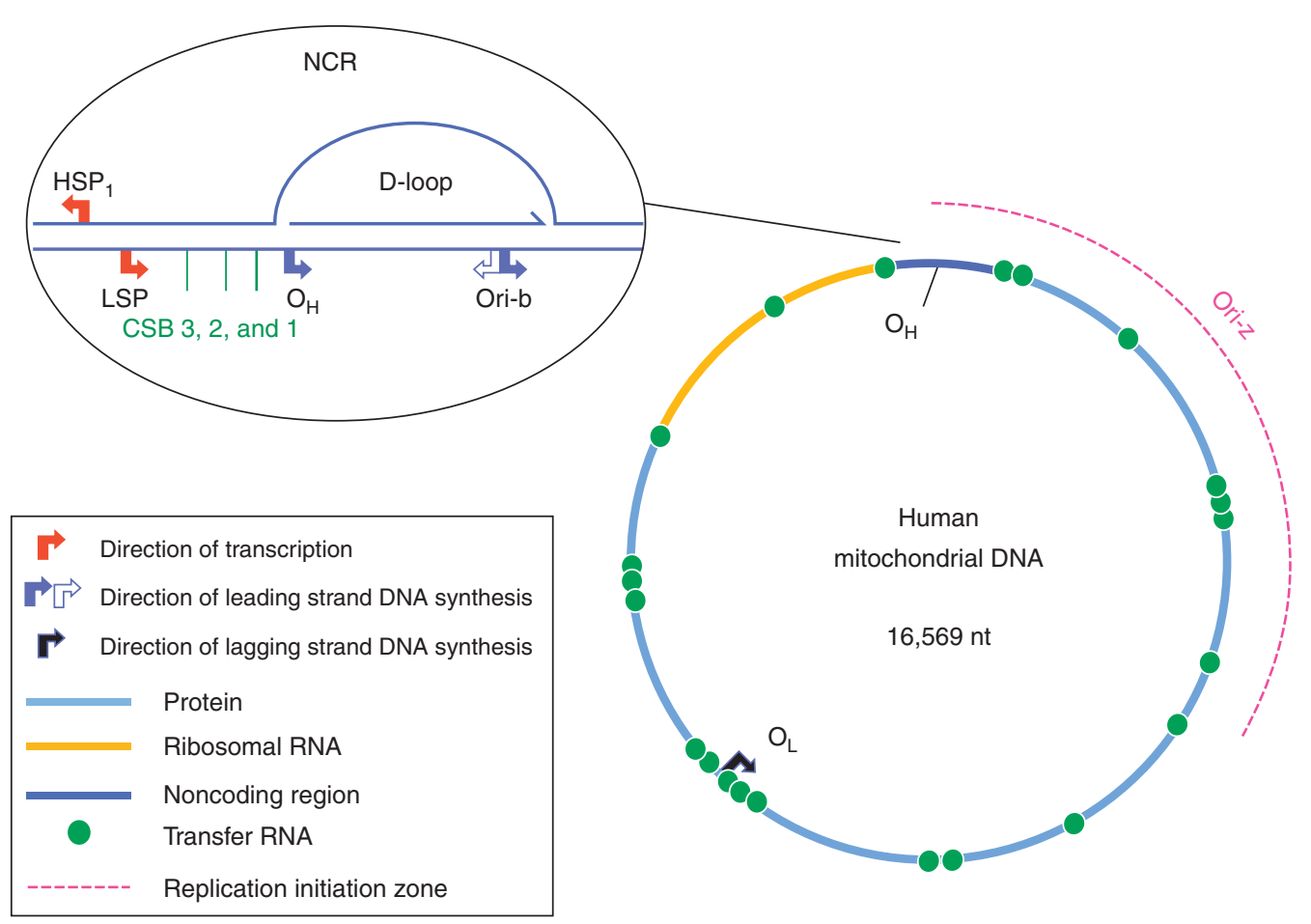

Figure 1. Schematic diagram of the human mitochondrial genome. Human mtDNA encodes 13 proteins that are essential for oxidative phosphorylation, and the ribosomal and transfer RNAs necessary for their translation. RITOLS replication initiates in the major noncoding region, at $\mathrm{O}_{\mathrm{H}}$ and Ori-b, and $\mathrm{O}_{\mathrm{L}}$ is a major initiation site of lagging strand DNA synthesis in mammals. Conversely, conventional bidirectional replication of mtDNA can initiate almost anywhere in the mitochondrial genome, with most such events occurring in a region of several kilobases adjacent to the major noncoding region (NCR), Ori-z (see main text for details). LSP, light strand promoter; HSP, heavy strand promoter; CSBs, conserved sequence blocks.

occupies much but not all, of the NCR, and so the terms are not interchangeable. The NCR is also known as the control region as it contains important cis-elements for transcription and two putative origins of replication. The critical importance of the NCR to genome maintenance is attested to by the fact that all, of the many, partially deleted mtDNAs that have been characterized in humans retain the NCR (Samuels et al. 2004), although at least a quarter of the D-loop region appears to be dispensable (Behar et al. 2008).

Many researchers date the discovery of DNA in mitochondria to the 1960s, when Nass published electron micrograph images of structures with the staining properties of DNA in chicken liver mitochondria, and Schatz isolated DNA from yeast mitochondria (Nass and Nass 1963;
Schatz 1963); yet the existence of mitochondrial DNA (mtDNA), was inferred much earlier, when some heritable traits were shown to be transmitted via the cytoplasm, rather than the nucleus (Ephrussi 1953; Caspari 1955).

\section{MECHANISMS OF MITOCHONDRIAL DNA REPLICATION}

\section{Strand-Displacement Replication}

A mechanism of replication for mammalian mtDNA was elaborated in the early 1970s from EM images of DNA isolated from rodent mitochondria by cesium chloride density centrifugation (Kasamatsu and Vinograd 1973). The mechanism is quite different from that of bacterial chromosomal replication and DNA 
replication in the nucleus, as almost all the "theta"-like molecules had several kilobases of single-stranded DNA on one branch. Thus, the EM images of mouse mtDNA suggested a model whereby leading strand synthesis started at a specific site and advanced approximately twothirds of the way around the molecule before second-strand DNA synthesis initiated (Kasamatsu and Vinograd 1973). The mechanism has enjoyed a number of names: asynchronous, asymmetric, and strand-displacement replication, but none is entirely satisfactory: uncoupled (leading and lagging strand DNA synthesis) replication is perhaps a more informative name, but we will continue to employ the acronym SDM (strand-displacement mechanism), for reasons of continuity. In apparent support of SDM, free $5^{\prime}$ ends of DNA, were mapped $\sim 11$ kilobases apart and on opposite strands (Clayton 1982). These are candidate initiation sites for leading $\left(\mathrm{O}_{\mathrm{H}}\right)^{1}$ and lagging $\left(\mathrm{O}_{\mathrm{L}}\right)^{1}$ strand DNA synthesis (see Fig. 1), although as discussed below free $5^{\prime}$ ends of DNA are not necessarily synonymous with replication initiation sites. Although starkly different from nuclear DNA replication, SDM was not unprecedented, as several plasmids and viruses have a protracted delay between the initiation of leading and lagging strand DNA synthesis (Khan 1997).

David Wolstenholme and colleagues produced electron micrographs of replicating molecules of rat liver mtDNA that were fully duplex, as long ago as the late 1960s (Kirschner et al. 1968), although the same group later reported that other preparations of mammalian mtDNA contained many partially single-stranded replicating molecules, leading them to propose a step-wise mode of replication, in addition to SDM. In the former case, repeated pausing of leading strand DNA synthesis at approximately $2.5 \mathrm{~kb}$ intervals acts as the prelude to the initiation of second-strand DNA synthesis (Koike and

\footnotetext{
${ }^{1}$ The two strands of mitochondrial DNA have different buoyant densities on denaturing caesium chloride gradients because of differences in their nucleotide composition and so they are denoted $\mathrm{H}$ (heavy) and L (light) strands; the putative initiation sites were named the heavy and light strand origins of replication $\left(\mathrm{O}_{\mathrm{H}}\right.$ and $\mathrm{O}_{\mathrm{L}}$, respectively), accordingly.
}

Wolstenholme 1974; Wolstenholme et al. 1974). Today we would recognize this as a potential strand-switching mode of replication (Michel et al. 2004). The fact that other articles from Wolstenholme's own group emphasized the novelty of the partially single-stranded intermediates of replicating mtDNA (e.g., Wolstenholme et al. 1974), doubtless contributed to the fully duplex species being largely ignored for three decades. Moreover, SDM was elaborated in some detail over the course of the next decade (Clayton and Larsson 2006, and references therein), whereas no new data emerged to support the idea of multiple sites of initiation on the lagging strand. Thus, it wasn't until restriction digested human and rodent mtDNAwere shown to form arcs of replication intermediates characteristic of fully duplex DNA, using neutral two-dimensional agarose gel electrophoresis (2D-AGE), that the dominance of the strand-displacement model was seriously challenged (Holt et al. 2000). 2D-AGE resolves DNA molecules on the basis of a combination of shape and mass; aficionados of DNA replication love the method, because it captures the entire process and provides detailed mechanistic insight (Brewer and Fangman 1987, 1991; Friedman and Brewer 1995). However, it is generally loathed by the wider world because it takes considerable time and effort to learn to interpret the complex arcs and spots to which the method gives rise: it says it all that the children of one of the investigators referred to the autoradiographs of replicating mtDNA scattered about the house as the "squashed-fly pictures." Actually, the arcs comprise a complete series of intermediates from all stages of the replication cycle. Fortunately, for nonspecialists and specialists alike, some nucleic acid modifying enzymes dramatically alter the patterns of replication intermediates (RIs), greatly aiding interpretation. In the first such study of mammalian mtDNA replication (Holt et al. 2000), the use of single-stranded nuclease was especially helpful as it degraded all partially single-stranded species (Fig. 2); thus, the surviving mitochondrial RIs did not simply have the shape and mobility of fully duplex intermediates, they proved resistant to enzymes that degrade single-stranded DNA. In mitochondria 
I.J. Holt and A. Reyes

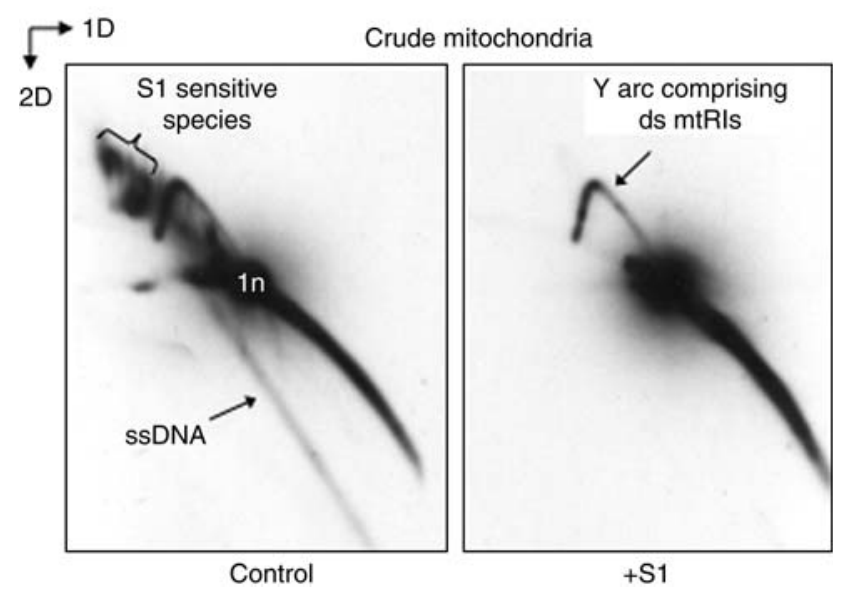

Figure 2. Some mitochondrial replication intermediates form standard replication fork arcs and are resistant to single-strand nuclease. In the example shown mouse liver mtDNA was digested with DraI and half the digested was additionally treated with S1 nuclease prior to 2D-AGE. After blotting the DraI filter was hybridized with probe that detected a $4.5 \mathrm{~kb}$ fragment of mouse mtDNA, np 5 276-9 817. ssDNA, single-stranded DNA; ds mtRIs, double-stranded mitochondrial replication intermediates.

of human cells and placenta, and mouse liver, the signal along the length of many of the replication arcs was sufficiently even to exclude not only SDM, but also the regimented step-wise replication model of Wolstenholme (Koike and Wolstenholme 1974). Instead, the single-strand nuclease resistant mtRIs were proposed to be the products of coupled leading and lagging strand DNA synthesis (Holt et al. 2000), which is discussed in more detail in the next section but one.

\section{RNA INCORPORATION DURING MTDNA REPLICATION: RITOLS}

The presumed products of strand-coupled DNA synthesis appeared to be in the minority, relative to the partially single-stranded species, and the latter were at that time attributed to SDM (Holt et al. 2000). However, later more highly purified mitochondria yielded unusually large DNA molecules forming slow-moving arcs of replication intermediates, in place of the partially single-stranded species (Fig. 3A) (Yang et al. 2002). The components of the slow-moving arcs corresponded to molecules that the restriction enzymes were unable to cut at one or more sites on one branch. ${ }^{2}$ At first inspection, the phenomenon of the slow-moving arcs appeared compatible with SDM, because most restriction enzymes cannot cut single-stranded DNA. However, several other features and properties of the slow-moving arcs could not be reconciled with SDM. Based on their masses, the species of the slow-moving arcs were fully duplex (Fig. 3A) and (Yasukawa et al. 2006). A more clear-cut distinction was provided by the few restriction endonucleases that can cut single-stranded, as well as duplex, DNA. Crucially, such enzymes would not produce slow-moving replication fork arcs in the case of SDM, instead they would create arcs that begin at the usual point immediately adjacent to nonreplicating fragments of DNA (termed 1n species) but end prematurely, at $1.5 \mathrm{n}$ rather than the usual $2 \mathrm{n}$. In practice, digesting gradient-purified mtDNA with enzymes that cut both duplex and single-stranded DNA gave rise to the same slow-moving Yarcs, as

\footnotetext{
${ }^{2}$ Enzymes that cut a replicating molecule on both branches produce so-called replication fork, or Y arcs, enzymes cutting neither branch will yield bubble structures; in the case of mtDNA, enzymes were frequently cutting only one branch of the bubble (Yasukawa et al. 2006 and Figure 3).
} 
Human Mitochondrial DNA Replication

A

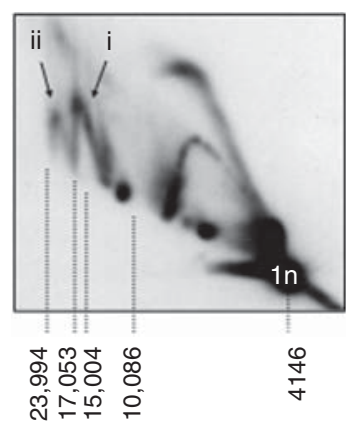

B
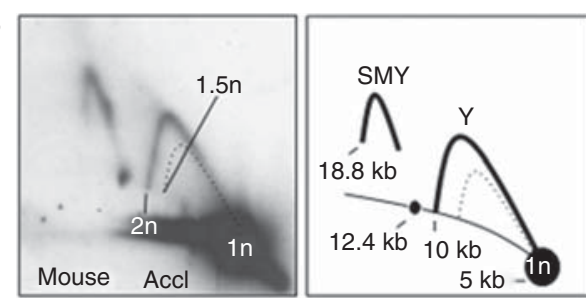

C
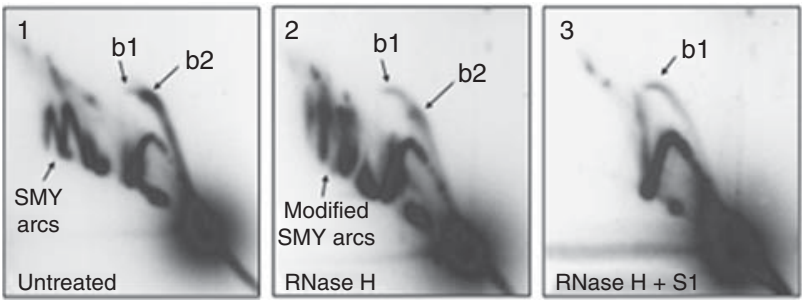

Figure 3. Replicating molecules of mtDNA produce duplex slow-moving arcs, which are sensitive to RNase H. (A) 2D-AGE of BclI digested mouse liver mtDNA reveals a pair of slow-moving replication fork (SMY) arcs, after hybridization to a probe detecting the $4 \mathrm{~kb}$ fragment nucleotides 12,027-16,174. SMY arcs result from blocked restriction sites and are essentially duplex, based on their mass, arc i has a theoretical mass of 13,096 bp initially, increasing to $17,341 \mathrm{bp}$ if fully duplex, whereas it would begin at 10,744 and end at 12,866 bp if it contained a single-stranded DNA branch. Arc ii spans 17,078-21,060 if duplex; 12,603-14,594 if it follows SDM. The spans of the arcs predicted by SDM are indicated in gray font, as no such arcs were detected when gradient-purified mtDNA was analyzed. (B) 2D-AGE of AccI digested mouse liver mtDNA reveals a slow-moving replication fork (SMY) arc, after hybridization to a probe detecting the 5-kb fragment, nucleotides 9602-14,654. N.B., AccI cleaves both single-stranded and double-stranded DNA. Dashed line corresponds to the expected arc in the case of SDM replication. (C) The same digest of purified mouse liver mtDNA as shown in panel Awas incubated with and without RNase $\mathrm{H}$ and single-stranded (S1) nuclease, and analyzed by 2D-AGE. SMYarcs are inferred to have ribonucleotides at one or more restriction sites, in part because the SMY arcs are grossly modified by RNase H. Similarly, bubble arc b2 is also sensitive to RNase $\mathrm{H}$ and therefore contains significant amounts of RNA, as opposed to bubble arc b1; see also main text and Yasukawa et al. (2006) for details.

enzymes that cannot cut single-stranded DNA (Fig. 3B) (Yasukawa et al. 2006). If the novel arcs were not partially single-stranded, what then could be responsible for these unusual species? Based on their mass and shape they were indistinguishable from duplex DNA; nevertheless, they were not cut by REs on one branch. Because the samples had been treated thoroughly with protease and phenol-chloroform extracted, sometimes repeatedly, protein was unlikely to account for the slow-moving arcs. Lipid and carbohydrate seemed even more improbable, and so that left RNA.

Nature had thoughtfully provided the precise tool to test this hypothesis. RNase $\mathrm{H}$ degrades RNA only if it is hybridized to DNA, 
I.J. Holt and A. Reyes

and it proved highly effective at modifying mitochondrial RIs (Fig. 3C) (Yang et al. 2002). The presence of RNA complementary to the lagging strand template was confirmed by extracting mtRIs from $2 \mathrm{D}$ gels, subjecting them to denaturation, $1 \mathrm{D}-\mathrm{AGE}$ and hybridization to reveal Lstrand RNAs of 200-600 nucleotides in length (Yasukawa et al. 2006). Therefore, we proposed that RNA was incorporated throughout the lagging strand during the replication of mtDNA: the RITOLS model of mtDNA replication (Fig. 4). The source of the RNA has not yet been established, but two possible models were mooted: the RNA might be the product of an exuberant primase, synthesizing long RNA "primers," or the RNA could be derived from preformed transcripts which were threaded onto the lagging strand template, the so-called bootlace model (Yasukawa et al. 2006).

The RITOLS model of replication has much in common with the SDM. Both models predict that the two strands of DNA are not synthesized concurrently and both mark " $\mathrm{O}_{\mathrm{L}}$ " as a major initiation site of second-strand DNA synthesis in mammals. Strip away the RNA from RITOLS intermediates and one is left with molecules that conform, to a first approximation, to intermediates of SDM. Or put another way SDM + lagging strand RNA = RITOLS replication. The RITOLS mechanism also retains the major noncoding region (NCR) as the origin-containing region, although there appear to be not one, but two origins in this region (Yasukawa et al. 2006), roughly speaking at either end of the D-loop.

One potential advantage of RITOLS over SDM is avoiding exposing direct repeats as single-stranded DNA, which in bacterial plasmids increases the frequency of deletion formation (Bron et al. 1991). Many pathological partial deletions of mtDNA have a residual direct repeat at the deletion junction (Mita et al. 1990), and these might be much more frequent without RNA to protect the lagging strand template. It has been suggested that the truth may lie

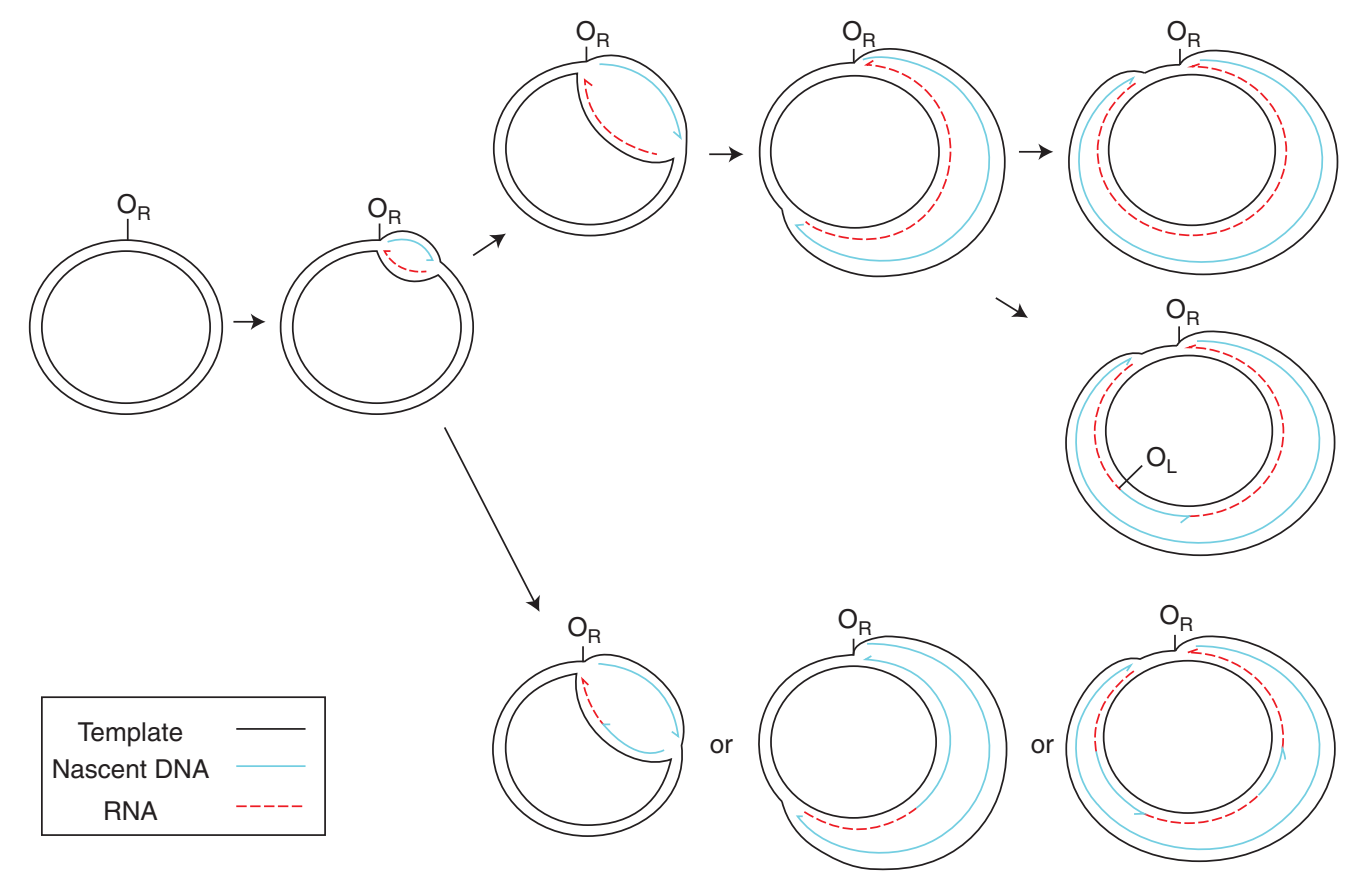

Figure 4. The RITOLS model of mitochondrial DNA replication. Replication initiates at one of two sites $\left(\mathrm{O}_{\mathrm{H}}\right.$ or Ori-b), here conflated to $\mathrm{O}_{R}$, for origin of replication. Leading strand DNA synthesis progresses with concurrent incorporation of RNA on the lagging strand. At some point (frequently $\mathrm{O}_{\mathrm{L}}$ ), lagging strand DNA synthesis initiates and the lagging strand RNA is replaced by, or converted to, DNA. 
somewhere between the SDM and the RITOLS models; i.e., some sections of the lagging strand template might be hybridized to RNA, whereas other sections might be coated with the mitochondrial single-stranded binding protein (mtSSB) (Wanrooij and Falkenberg 2010). Of course it would come as no surprise if there were some $\mathrm{mtSSB}$ at the replication fork, as per conventional strand-coupled DNA replication, yet the data firmly predict that RNA coats much, if not essentially all, of the lagging strand. First, the slow-moving arcs accounted for the same proportion of mitochondrial RIs irrespective of an enzyme's ability to cut ssDNA; second, the masses of the mitochondrial RIs corresponded to fully duplex DNA, within the limits of the technique (Fig. 3A) (Yang et al. 2002; Yasukawa et al. 2006). Thus, we estimate RNA coverage of the lagging strand to be at least $80 \%$ based on 2D-AGE analysis. More recent EM images revealed no gaps whatsoever in the vast majority of replicating molecules of mtDNA (Pohjoismaki et al. 2010). This result suggests that single-stranded regions account for no more than 100-200 nucleotides of mtDNA. There is a good reason to expect the coverage to be all but total; RNA has a major theoretical advantage over protein. Although the fidelity of RNA synthesis is lower than DNA synthesis, RNA and DNA contain the same genetic information, and so RNA could be used to repair any damage that occurs to the lagging strand template during mtDNA replication; no protein could perform this role. If this is the reason that RNA is preferred to $\mathrm{mtSSB}$, the entire displaced lagging strand template will be hybridized to RNA. Ironically, given the predictions of extensive single-strandedness that are inherent to SDM, RNA incorporation affords the possibility of having even less single-strandedness than conventional replication mechanisms, if it follows the aforementioned bootlace model. This is because the use of preformed transcripts would dispense with the need to loop out the lagging strand for Okazaki fragment synthesis. If true, then this might explain why RITOLS evolved, as it could reduce the risk of DNA damage imposed by the formation of single-stranded DNA at the replication fork. This may be critically important in the cru- cible of the mitochondrion, in which reactive oxygen species are all too frequent by-products of respiration. Alternatively or additionally, RITOLS may be designed to prevent the deleterious consequences of collisions between transcription and replication complexes (Pomerantz and O'Donnell 2010), either by inhibiting transcription altogether on replicating molecules (Bentin et al. 2005) or to reduce the speed of replication, thereby, quite literally, reducing the impact of any such collisions.

Why then hasn't RITOLS been more widely adopted? It may well be that it is simply too slow-mtDNA replication being notoriously laggardly, at least in cell culture (Bogenhagen and Clayton 1977). Moreover, if RITOLS uses preformed transcripts (the bootlace model) then it could only be adopted for DNA sequences that are constitutively transcribed. This would exclude many bacterial genomes in which different genes are expressed under different conditions, which would create gaps were RITOLS replication to be used, and the nuclear genome, which comprises mainly noncoding sequences. Nevertheless, it might be worthwhile to inspect closely the replication of nuclear ribosomal DNA genes. The long lengths of noncoding DNA in plant mtDNAs also suggest that it will eschew RITOLS replication, for the most part.

\section{COUPLED LEADING AND LAGGING STRAND DNA SYNTHESIS}

The first 2D-AGE study of mammalian mtDNA replication concluded that a proportion of mitochondrial RIs comprised fully duplex DNA and so they were proposed to be the products of coupled leading and lagging strand DNA replication (Holt et al. 2000). This was a tentative conclusion, although it did suggest as a minimum that second-strand DNA synthesis could initiate at many positions on the circle. A later study (Bowmaker et al. 2003) provided further insight, yet was handicapped by the fact that the RITOLS mechanism (Yasukawa et al. 2006) had yet to be elaborated. For the benefit of readers, we will attempt to reconstruct events with the benefit of hindsight. The 2003 study mapped the majority of replication initiation sites to a zone 
of several kilobases outside the NCR, suggesting that initiation of replication rarely starts at the widely recognized unidirectional origin, $\mathrm{O}_{\mathrm{H}}$. In apparent contradiction of these results, the 2006 study mapped the start of replication to two tightly defined areas both within the NCR, one of which was $\mathrm{O}_{\mathrm{H}}$. The explanation for this apparent discrepancy is that the two reports delineated two different mechanisms of replication, the later one earmarking the NCR as the exclusive start region for RITOLS replication (Fig. 4). In contrast, the 2003 report (Bowmaker et al. 2003) characterized fully duplex DNA intermediates, the presumed products of coupled leading and lagging strand DNA synthesis, although nowhere is this explicitly stated in the manuscript for the aforementioned reasons. Another contrasting feature between the intermediates of RITOLS replication and strand-coupled DNA synthesis is directionality: RITOLS is substantially unidirectional (2006), whereas the replication initiation zone gave rise to bidirectional replication forks (Bowmaker et al. 2003). Further evidence of bidirectional replication of mtDNA came from the study of mitochondrial RIs of birds (Reyes et al. 2005). Unfortunately, this conclusion relied on an understanding of fork-direction gels (Friedman and Brewer 1995), whose interpretation is even more challenging than the standard 2D gel system. In essence, an in-gel digestion step trims the replication forks at one or other end, which generates one of two modified arcs, dependent on whether the fork entered the fragment at one end or the other-predicting the effects of different in-gel digests makes a great parlor game that all the family can enjoy! Based on a series of such digests, we were able to establish that replication forks enter many fragments of mtDNA from either end, again indicating that replication initiation occurs across a broad zone of several kilobases. Thus, both birds and mammals operate bidirectional strand-coupled DNA replication, initiating across a broad initiation zone (Reyes et al. 2005).

Currently the replication initiation zone is the only firm evidence we have for conventional coupled leading and lagging strand DNA synthesis in mitochondria, because mitochondrial RIs with duplex DNA on all branches can be derivatives of RITOLS replication. This was highlighted by the use of that most popular of drugs among mtDNA researchers, ethidium bromide. Without going into detail, ethidium bromide accumulates in mitochondria, and so low doses inhibit mtDNA replication, with little effect on nuclear DNA. Hence, three days exposure of growing cells to $125-\mu \mathrm{M}$ ethidium bromide results in a tenfold decrease in mtDNA copy number, with no change in the generation time. Removal of the drug is followed by a burst of mtDNA replication to restore copy number to normal. Harvesting mtDNA during the recovery phase revealed fully duplex mitochondrial RIs, which were suggestive of coupled leading and lagging strand DNA replication originating at a bidirectional origin within the NCR (Yasukawa et al.2005). At the time, we suggested this could be the result of either a contraction of the previously defined initiation zone, or rapid conversion of the "RITOLS" RNA to DNA (Fig. 5A) (Yasukawa et al. 2005). The rapid conversion of RNA to DNA, or early maturation hypothesis was strengthened when initiation of RITOLS replication was mapped to two sites in the NCR (Yasukawa et al. 2006), one of which corresponded to the novel origin identified in the ethidium bromide study (see below for more on origins of replication). It is probably the case that residual ethidium bromide causes stalling of leading strand DNA synthesis and that stalling promotes initiation of second-strand DNA synthesis. If this were to occur via strand-switching, it would breath fresh life into the early Wolstenholme model of mtDNA replication (Koike and Wolstenholme 1974). It is also noteworthy that mutant forms of the mitochondrial DNA helicase, Twinkle, produce substantially the same effect on the pattern of mtRIs as recovery from transient depletion, although the stalling is so severe that it causes mtDNA depletion (Wanrooij et al. 2007; Goffart et al. 2009).

\section{LET US START AT THE VERY BEGINNING}

The initiation of replication must necessarily create a piece of nucleic acid with a free $5^{\prime}$ end; typically this is the first base of a short RNA primer, which is conjugated to DNA. The RNA 

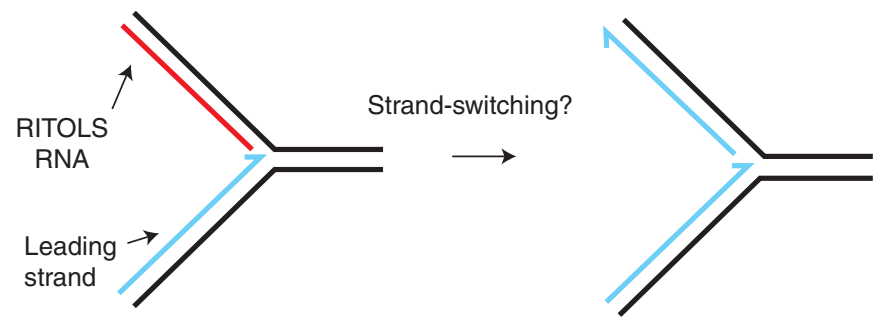

B

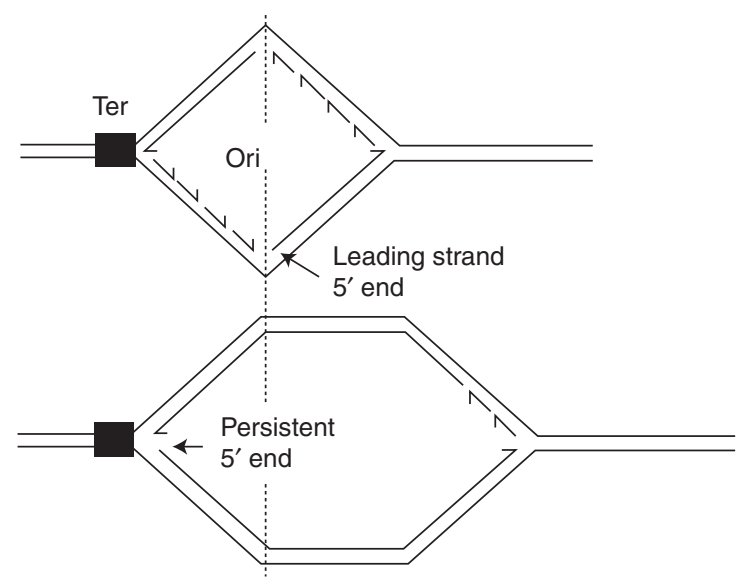

Figure 5. Maturation of RITOLS RNA, and the formation of free $5^{\prime}$ ends of DNA during DNA replication. Replication stalling is associated with an increase in fully duplex DNA intermediates, which could be the result of RNA replacement with DNA $(A)$. This could be the result of strand-switching by the replicative DNA polymerase. $(B)$ One of a pair of replication forks derived from a bidirectional origin of replication has just reached the terminus (Ter) causing arrest of leading strand DNA synthesis; the Okazaki fragments are joined to make the lagging strand continuous, leaving a single free $5^{\prime}$ end remaining at the terminus, whereas the origin is no longer detectable.

primer is chemically more labile than DNA and so it may degrade during nucleic acid extraction and processing. Even if the RNA primer is preserved, many DNA polymerases will not read through RNA, therefore primer extension can be used to map free $5^{\prime}$ ends of DNA, and this procedure was for many years the method of choice for defining origins of replication. In this way, the origin of replication of mtDNA was mapped to specific nucleotides in the NCR, especially nucleotide 191 of human mtDNA, aka $\mathrm{O}_{\mathrm{H}}$ (Crews et al. 1979). Later RNA species that spanned the region from the light strand promoter (LSP) to, approximately, $\mathrm{O}_{\mathrm{H}}$ were identified, leading to the suggestion that transcripts initiated at LSP could be processed to generate primers for the initiation of DNA synthesis, and so RNA processing of L-strand transcripts would be the first critical step in the initiation of mtDNA replication (Chang et al. 1985). It has been suggested that a catalytic (7-2/MRP) RNA is responsible for this RNA processing step (Topper et al. 1992), although there appears to be little if any 7-2/MRP in some mitochondrial preparations (Kiss and Filipowicz 1992).

Many features of this model may prove to be correct, but in light of recent history in the field, it is worthwhile considering several caveats. First, there are abundant 7S DNA molecules of 
mitochondrialD-loops, the $5^{\prime}$ ends of these molecules map to a cluster of nucleotides in the NCR including nucleotide 191. Ordinarily, they are far more abundant than RIs, and so (labeled) primers should be placed outside the NCR to be confident that they are not contributing to the results. Second, $5^{\prime}$ ends of DNA need not necessarily mark an initiation site: persistent nicks will give the same output. Third, origins dispersed across a zone of several kilobases will produce variable $5^{\prime}$ ends of DNA, which will almost certainly be below the limits of detection because, fourth, initiation sites of bidirectional replication are ephemeral creatures (Fig. 5B). Fifth, a free $5^{\prime}$ end of DNA may mark a replication pause or termination site, both of which will generate far more prominent $5^{\prime}$ ends of DNA than even a discrete origin of replication (Fig. 5B) (Santamaria et al. 1998; Hyvarinen et al. 2007), unless, that is, it is a unidirectional origin of replication.

Currently there are several candidate initiation sites for leading strand mtDNA synthesis. Early reports agreed that the most abundant free 5 ' ends of DNA lay at or close to nucleotide 191 of human mtDNA (e.g., Crews et al. 1979). Much later, Giuseppe Attardi revisited the question, and concluded that the major start site was a hundred or so nucleotides downstream of nucleotide 191, at nucleotide 57 (Fish et al. 2004). In contrast, Falkenberg and colleagues RNase $\mathrm{H}$ treated their samples and found prominent primer extension products mapping upstream of nucleotide 191, at the second of three conserved sequence blocks in the NCR, CSB2 (i.e., $\sim$ nucleotide 300) (Pham et al. 2006). Because the sites identified in these studies were all in the vicinity of one another they were all compatible with the early EM data (Kasamatsu and Vinograd 1973). Our mapping of free $5^{\prime}$ ends of DNA relied on ligation-mediated PCR, which we readily acknowledge is far from perfect, as the amplification process is at best semi-quantitative. However, the fine mapping was coupled to 2D-AGE analysis. The bubble arcs on 2D gels, which are a signature of replication origins, mapped a "RITOLS" origin (or terminus) approximately to $\mathrm{O}_{\mathrm{H}}$, and LM-PCR revealed free $5^{\prime}$ ends of DNA at nucleotides 54 and 191 (as well as a few points in between); although we did not detect ends at CSB2, our samples were not treated with RNase $\mathrm{H}$. No final ruling can be made as to the precise location of $\mathrm{O}_{\mathrm{H}}$, but there is little doubt an origin (and terminus) is located in the vicinity of nucleotide 191 . The second origin in the NCR revealed by the ethidium bromide study (Yasukawa et al. 2005) maps to the other end of the D-loop, around nucleotide 16,197 (aka Ori-b). This discovery raised the intriguing possibility that Ori-b may be the major or primary origin, and $\mathrm{O}_{\mathrm{H}}$ is the replication terminus. In this model, bidirectional replication from Ori-b produces a nascent leading strand of DNA that encounters the terminus after a mere half a kilobase. Concurrent Okazaki fragment synthesis fills in on the lagging strand template back to Ori-b (Fig. 5), and so the newly synthesized $\mathrm{H}$-strand DNA from $\mathrm{O}_{\mathrm{H}}$ to Ori-b is technically lagging strand, not leading strand, DNA. However, when RNA is incorporated on the L-strand template, the same piece of nascent $\mathrm{H}$-strand DNA $\left(\mathrm{O}_{\mathrm{H}}-\mathrm{Ori}-\mathrm{b}\right)$ cannot be said to be lagging strand, it then becomes a second initiation event on the leading strand; notwithstanding, this remains a subsidiary event to initiation at Ori-b.

\section{THE ENDGAME}

Almost nothing is known about the termination of mtDNA replication beyond its location. Terminal replication intermediates contain two forks, one or both of which is advancing toward the other, creating so-called double Yarcs on 2D gels. When the two forks meet, the molecule mimics a four-way junction (or X structure), before separating into the two daughter molecules. In most cells and tissues, double $\mathrm{Y}$ arcs of mtDNA are the exclusive preserve of fragments that contain the NCR, and the termination site maps to $\mathrm{O}_{\mathrm{H}}$. In bacteria, termination of replication and resolution of replication intermediates is a well-orchestrated process, which requires a site-specific recombination apparatus (Grainge et al. 2011). To date, we merely have the suggestion that three proteins of the mTERF family, mTERF, mTERFD1, and mTERFD3, in some way contribute to the termination of DNA 
replication. Overexpression of $\mathrm{mTERF}$ induces an increase in $5^{\prime}$ ends at $\mathrm{O}_{\mathrm{H}}$ without altering mtDNA copy number (Hyvarinen et al. 2007), which is suggestive of prolonged termination (Pohjoismaki and Goffart 2011). And gene-silencing of mTERFD1 and mTERFD3 leads to the accumulation of $\mathrm{X}$ species, centered on $\mathrm{O}_{\mathrm{H}}$ (Hyvarinen et al. 2011).

\section{THE MACHINERY OF MITOCHONDRIAL DNA REPLICATION: WHERE'S THE BEEF?}

Remarkably few enzymes are known to be critical for mtDNA replication in mammals, those known or inferred to be essential are mitochondrial DNA polymerase $\gamma$ (Hance et al. 2005), Twinkle DNA helicase (Tyynismaa et al. 2004), mitochondrial RNA polymerase, single-stranded DNA binding protein, RNase H1 (Cerritelli et al. 2003), DNA ligase III (Simsek et al. 2011), and topoisomerases. We confidently predict that this is very far from a full list, despite the fact that the first two, with mtSSB and TFAM, can synthesize pieces of DNA up to 16 kilobases in length, in vitro (Korhonen et al. 2004).

If conventional strand-coupled DNA replication occurs in mitochondria then there must be the apparatus for Okazaki fragment maturation, the sealing of the discontinuous segments of DNA that comprise the nascent lagging strand of DNA. One firm possibility is that the enzymes involved are shared with the nucleus (Holt 2009). PIF1 (Futami et al. 2007), FEN1 (Liu et al. 2008), and DNA2 (Duxin et al. 2009) have each been reported to be present in mitochondria, as well as in the nucleus, but the last two might be used exclusively in DNA repair, equally PIF1 could have functions unrelated to DNA replication. Nevertheless, it is noteworthy that all the apparatus of Okazaki fragment maturation appears to be present in mitochondria — it would be a pity to ignore it.

If, as the data suggest, mitochondria operate two, or more, mechanisms of replication then there is no reason a priori why they should restrict themselves to a single DNA polymerase. We forgot at our peril that the field of bacterial DNA replication blithely believed pol I was the replicative DNA polymerase for 15 years. More- over, mitochondria of Trypanosomes have four DNA polymerases (Klingbeil et al. 2002) and while it is true they have a bizarre arrangement of mtDNA, we doubt that more sophisticated mammals ration themselves to a single enzyme, given the importance of the mitochondrial genome.

\section{REPLICATION AND SELECTION OF MUTANT MITOCHONDRIAL DNA}

Interest in mitochondrial DNA replication stems in part from the human diseases caused by mutant mtDNA. Pathological mutations in human mtDNA were first described early in 1988; these were partial deletions that resulted in the loss of multiple genes (Holt et al. 1988). A key distinction from nuclear DNA mutations is that mutant mtDNA does not follow Mendelian genetics, both because mtDNA is maternally inherited and because there are typically thousands of copies of mtDNA per cell. Thus, a variant of mtDNA may account for anywhere between $0.1 \%$ and $99.9 \%$ of the mitochondrial genotype. In most individuals, the vast majority of mtDNA molecules share the same sequence (homoplasmy), whereas deleterious mutants often coexist with wild-type molecules (heteroplasmy). Clearly this suggests competition between different mitochondrial genotypes. $\mathrm{Mu}$ tants, such as partial deletions, that result in complete loss of function can never achieve total dominance (homoplasmy) in complex organisms, because aerobic energy production is essential for life, yet there is no question that mutant mtDNA can accumulate to levels that seriously impair respiration and produce devastating diseases, as was clear from the very first report (Holt et al. 1988). Although we are still grappling with the forces that drive selection of mutant mtDNA, the underlying problem of replicative advantage is becoming clear, at least in some cases. The danger for the cell is that any mutation that confers on mtDNA a replicative advantage will result in the selection of the mutant genotype irrespective of its effect on mitochondrial function.

What do we mean by the rather nebulous concept of replicative advantage? As has been 
widely recognized, partial deletions of mtDNA will complete the replication cycle ahead of their full-length, wild-type counterparts, and so they will be the first daughter molecules available for subsequent rounds of replication (Diaz et al. 2002). Partial duplications have more rather than less mtDNA to copy and so should have a marked disadvantage; however, this is more than compensated for if, as is invariably the case in patients, the extra DNA contains an additional origin(s) of replication (Holt et al. 1997). The point mutation most frequently associated with disease in humans resides at nucleotide position 3243 (Goto et al. 1990), far from any predicted origin of replication, yet it too is thought to confer a selective advantage to its owner. Nucleotide 3243 is located in the binding site for a protein known as the mitochondrial transcription termination factor, mTERF. Overexpression of mTERF enhances replication pausing around nucleotide 3243 (Hyvarinen et al. 2007). Crucially, the m.3243G mutation reduces the affinity of binding of mTERF (Hess et al. 1991), and so it is predicted to reduce replication pausing; hence, m.3243G can confer a replicative advantage. Although it is not clear if every one of the hundreds of pathological mtDNA mutations confers a direct replicative advantage, there is currently no other hypothesis to explain the preferential accumulation of deleterious mitochondrial variants. Although impaired mitochondrial clearance (mitophagy) could permit mitochondria containing mutant DNA to thrive, such a defect should result in any and every mutant mtDNA variant persisting, rather than the fixation of specific mutants, as is seen in mitochondrial diseases.

\section{NUCLEAR DNA MUTATIONS AFFECTING MITOCHONDRIAL DNA}

Mitochondrial DNA of humans and most other animals is entirely reliant on the nucleus for its existence. This is amply shown by the many disease-causing mutations in genes required for mtDNA maintenance: POLG1, Twinkle (Spelbrink et al. 2001; Van Goethem et al. 2001). However, this is not a reliable method of assessing a protein's credentials for a role in replica- tion, or mtDNA maintenance. TFAM, which is absolutely essential for mtDNA maintenance (Larsson et al. 1998), has thus far never been linked to a human pathology. Moreover, if proteins are shared between the mitochondria and the nucleus many mutations will prove lethal because of their effects on nuclear DNA, or if they permit survival they may produce unusual phenotypes; that said, given the huge diversity of phenotypes associated with mitochondrial dysfunction it is hard to picture an atypical set of signs and symptoms.

\section{CONCLUDING REMARKS}

As the opening sentence of the abstract indicated, there is much work to be performed, and the full text should have left the reader in no doubt as to the many gaps in our knowledge, which are unlikely all to be filled by $\mathrm{mtSSB}$. The single most pressing issue is to confirm or refute the presence of RNA in mitochondrial replication intermediates, as this lies at the heart of the competing RITOLS and SD mechanisms. If confirmed, what is the source of the RITOLS RNA, and does it function to slow replication or inhibit transcription, as here proposed? Can mtDNA replication proceed via SDM in the absence of the RITOLS RNA, and if not, why not? Mitochondrial RNA polymerase appears to have a multiple roles in mtDNA replication, for example (Fuste et al. 2010), and further study of it, and the relationship between transcription and replication, are also likely to yield further insight into the mechanisms of mtDNA replication. We anticipate the identification of new enzymes involved in mtDNA replication will help enormously to unravel the mechanics of the process. As if two (or three?) mechanisms weren't enough, there is additionally evidence of recombination-dependent replication operating in some solid tissues of adults (Pohjoismaki et al. 2009), which awaits detailed investigation. Major topics such as replication restart and fork-rescue have barely been touched on in mitochondria, and even if the mechanics of initiation of leading strand DNA synthesis are as simple as some have suggested, we still know almost nothing of how it is regulated. 
Although the prospects for therapies that rely on intervening directly in the process of mtDNA replication seem bleak given the paucity of knowledge of the basic mechanisms, segregation bias may provide an indirect means of solving the problem. Also the burgeoning evidence of DNA transacting proteins shared between the nucleus and mitochondria should act as a stimulus for further research, and as previously noted, elucidation of mtDNA replication may even aid understanding of the same process in its giant neighbor, the nucleus (Holt 2009).

\section{ACKNOWLEDGMENTS}

We gratefully acknowledge the many colleagues and collaborators who have worked with us on mitochondrial DNA replication over the course of the past decade and a half, and the Medical Research Council and the European Union for funding. In addition, we thank Jaakko Pohjoismaki for a critical reading of the manuscript.

\section{REFERENCES}

Arnberg A, van Bruggen EF, Borst P. 1971. The presence of DNA molecules with a displacement loop in standard mitochondrial DNA preparations. Biochim Biophys Acta 246: 353-357.

Behar DM, Blue-Smith J, Soria-Hernanz DF, Tzur S, Hadid Y, Bormans C, Moen A, Tyler-Smith C, Quintana-Murci L, Wells RS. 2008. A novel 154-bp deletion in the human mitochondrial DNA control region in healthy individuals. Hum Mutat 29: 1387-1391.

Bentin T, Cherny D, Larsen HJ, Nielsen PE. 2005. Transcription arrest caused by long nascent RNA chains. Biochim Biophys Acta 1727: 97-105.

Bogenhagen D, Clayton DA. 1977. Mouse L cell mitochondrial DNA molecules are selected randomly for replication throughout the cell cycle. Cell 11: 719-727.

Bowmaker M, Yang MY, Yasukawa T, Reyes A, Jacobs HT, Huberman JA, Holt IJ. 2003. Mammalian mitochondrial DNA replicates bidirectionally from an initiation zone. J Biol Chem 278: 50961-50969.

Bratic I, Hench J, Henriksson J, Antebi A, Burglin TR, Trifunovic A. 2009. Mitochondrial DNA level, but not active replicase, is essential for Caenorhabditis elegans development. Nucleic Acids Res 37: 1817-1828.

Brewer BJ, Fangman WL. 1987. The localization of replication origins on ARS plasmids in S. cerevisiae. Cell 51: 463-471.

Brewer BJ, Fangman WL. 1991. Mapping replication origins in yeast chromosomes. Bioessays 13: 317-322.
Bron S, Holsappel S, Venema G, Peeters BP. 1991. Plasmid deletion formation between short direct repeats in Bacillus subtilis is stimulated by single-stranded rolling-circle replication intermediates. Mol Gen Genet 226: 88-96.

Caspari E. 1955. The role of genes and cytoplasmic particles in differentiation. Ann NY Acad Sci 60: 1026-1037.

Cerritelli SM, Frolova EG, Feng C, Grinberg A, Love PE, Crouch RJ. 2003. Failure to produce mitochondrial DNA results in embryonic lethality in Rnaseh1 null mice. Mol Cell 11: 807-815.

Chang DD, Hauswirth WW, Clayton DA. 1985. Replication priming and transcription initiate from precisely the same site in mouse mitochondrial DNA. EMBO J 4: 1559-1567.

Clayton DA. 1982. Replication of animal mitochondrial DNA. Cell 28: 693-705.

Clayton DA, Larsson NG. 2006. Mitochondrial DNA replication and human disease. In DNA replication and human disease (ed. Depamphilis ML), pp. 547-560. Cold Spring Harbor Laboratory Press, Cold Spring Harbor, NY.

Crews S, Ojala D, Posakony J, Nishiguchi J, Attardi G. 1979. Nucleotide sequence of a region of human mitochondrial DNA containing the precisely identified origin of replication. Nature 277: 192-198.

Desjardins P, Frost E, Morais R. 1985. Ethidium bromideinduced loss of mitochondrial DNA from primary chicken embryo fibroblasts. Mol Cell Biol 5: 1163-1169.

Diaz F, Bayona-Bafaluy MP, Rana M, Mora M, Hao H, Moraes CT. 2002. Human mitochondrial DNA with large deletions repopulates organelles faster than full-length genomes under relaxed copy number control. Nucleic Acids Res 30: 4626-4633.

Duxin JP, Dao B, Martinsson P, Rajala N, Guittat L, Campbell JL, Spelbrink JN, Stewart SA. 2009. Human Dna2 is a nuclear and mitochondrial DNA maintenance protein. Mol Cell Biol 29: 4274-4282.

Ephrussi B. 1953. Nucleo-cytoplasmic relations in microorganisms. Clarendon, New York.

Fish J, Raule N, Attardi G. 2004. Discovery of a major Dloop replication origin reveals two modes of human mtDNA synthesis. Science 306: 2098-2101.

Friedman KL, Brewer BJ. 1995. Analysis of replication intermediates by two-dimensional agarose gel electrophoresis. Methods Enzymol 262: 613-627.

Fuste JM, Wanrooij S, Jemt E, Granycome CE, Cluett TJ, Shi Y, Atanassova N, Holt IJ, Gustafsson CM, Falkenberg M. 2010. Mitochondrial RNA polymerase is needed for activation of the origin of light-strand DNA replication. Mol Cell 37: 67-78.

Futami K, Shimamoto A, Furuichi Y. 2007. Mitochondrial and nuclear localization of human Pif1 helicase. Biol Pharm Bull 30: 1685-1692.

Goffart S, Cooper HM, Tyynismaa H, Wanrooij S, Suomalainen A, Spelbrink JN. 2009. Twinkle mutations associated with autosomal dominant progressive external ophthalmoplegia lead to impaired helicase function and in vivo mtDNA replication stalling. Hum Mol Genet 18: $328-340$.

Goldring ES, Grossman LI, Krupnick D, Cryer DR, Marmur J. 1970. The petite mutation in yeast. Loss of 
I.J. Holt and A. Reyes

mitochondrial deoxyribonucleic acid during induction of petites with ethidium bromide. J Mol Biol 52: 323335.

Goto Y, Nonaka I, Horai S. 1990. A mutation in the tRNA (Leu)(UUR) gene associated with the MELAS subgroup of mitochondrial encephalomyopathies. Nature 348: 651-653.

Grainge I, Lesterlin C, Sherratt DJ. 2011. Activation of XerCD-dif recombination by the FtsK DNA translocase. Nucleic Acids Res 39: 5140-5148.

Hance N, Ekstrand MI, Trifunovic A. 2005. Mitochondria DNA polymerase $\gamma$ is essential for mammalian embryogenesis. Hum Mol Genet 14: 1775-1783.

Hess JF, Parisi MA, Bennett JL, Clayton DA. 1991. Impairment of mitochondrial transcription termination by a point mutation associated with the MELAS subgroup of mitochondrial encephalomyopathies. Nature 351: 236-239.

Holt IJ. 2009. Mitochondrial DNA replication and repair: All a flap. Trends Biochem Sci 34: 358-365.

Holt IJ, Harding AE, Morgan-Hughes JA. 1988. Deletions of muscle mitochondrial DNA in patients with mitochondrial myopathies. Nature 331: 717-719.

Holt IJ, Dunbar DR, Jacobs HT. 1997. Behaviour of a population of partially duplicated mitochondrial DNA molecules in cell culture: Segregation, maintenance and recombination dependent upon nuclear background. Hum Mol Genet 6: 1251-1260.

Holt IJ, Lorimer HE, Jacobs HT. 2000. Coupled leading- and lagging-strand synthesis of mammalian mitochondrial DNA. Cell 100: 515-524.

Hyvarinen AK, Pohjoismaki JL, Reyes A, Wanrooij S, Yasukawa T, Karhunen PJ, Spelbrink JN, Holt IJ, Jacobs HT. 2007. The mitochondrial transcription termination factor mTERF modulates replication pausing in human mitochondrial DNA. Nucleic Acids Res 35: 6458-6474.

Hyvarinen AK, Pohjoismaki JL, Holt IJ, Jacobs HT. 2011. Overexpression of MTERFD1 or MTERFD3 impairs the completion of mitochondrial DNA replication. Mol Bio Rep 38: 1321-1328.

Ingman M, Kaessmann H, Paabo S, Gyllensten U. 2000 Mitochondrial genome variation and the origin of modern humans. Nature 408: 708-713.

Kasamatsu H, Vinograd J. 1973. Unidirectionality of replication in mouse mitochondrial DNA. Nat New Biol 241: 103-105.

Kasamatsu H, Robberson DL, Vinograd J. 1971. A novel closed-circular mitochondrial DNA with properties of a replicating intermediate. Proc Natl Acad Sci 68: 22522257.

Khan SA. 1997. Rolling-circle replication of bacterial plasmids. Microbiol Mol Biol Rev 61: 442-455.

King MP, Attardi G. 1989. Human cells lacking mtDNA: Repopulation with exogenous mitochondria by complementation. Science 246: 500-503.

Kirschner RH, Wolstenholme DR, Gross NJ. 1968. Replicating molecules of circular mitochondrial DNA. Proc Natl Acad Sci 60: 1466-1472.

Kiss T, Filipowicz W. 1992. Evidence against a mitochondrial location of the 7-2/MRP RNA in mammalian cells. Cell 70: $11-16$.
Klingbeil MM, Motyka SA, Englund PT. 2002. Multiple mitochondrial DNA polymerases in Trypanosoma brucei. Mol Cell 10: 175-186.

Koike K, Wolstenholme DR. 1974. Evidence for discontinuous replication of circular mitochondrial DNA molecules from Novikoff rat ascites hepatoma cells. J Cell Biol 61: $14-25$

Korhonen JA, Pham XH, Pellegrini M, Falkenberg M. 2004. Reconstitution of a minimal mtDNA replisome in vitro. EMBO J 23: 2423-2429.

Larsson NG, Wang J, Wilhelmsson H, Oldfors A, Rustin P, Lewandoski M, Barsh GS, Clayton DA. 1998. Mitochondrial transcription factor $\mathrm{A}$ is necessary for mtDNA maintenance and embryogenesis in mice. Nat Genet 18: 231-236.

Levings CS 3rd, Pring DR. 1976. Restriction endonuclease analysis of mitochondrial DNA from normal and Texas cytoplasmic male-sterile maize. Science 193: 158-160.

Liu P, Qian L, Sung JS, de Souza-Pinto NC, Zheng L, Bogenhagen DF, Bohr VA, Wilson DM III, Shen B, Demple B. 2008. Removal of oxidative DNA damage via FEN1-dependent long-patch base excision repair in human cell mitochondria. Mol Cell Biol 28: 4975-4987.

Michel B, Grompone G, Flores MJ, Bidnenko V. 2004. Multiple pathways process stalled replication forks. Proc Natl Acad Sci 101: 12783-12788.

Mita S, Rizzuto R, Moraes CT, Shanske S, Arnaudo E, Fabrizi GM, Koga Y, DiMauro S, Schon EA. 1990. Recombination via flanking direct repeats is a major cause of large-scale deletions of human mitochondrial DNA. Nucleic Acids Res 18: $561-567$.

Nass MM, Nass S. 1963. Intramitochondrial fibers with DNA characteristics. I. Fixation and electron staining reactions. J Cell Biol 19: 593-611.

Newton KJ, Coe EH. 1986. Mitochondrial DNA changes in abnormal growth (nonchromosomal stripe) mutants of maize. Proc Natl Acad Sci 83: 7363-7366.

Pham XH, Farge G, Shi Y, Gaspari M, Gustafsson CM, Falkenberg M. 2006. Conserved sequence box II directs transcription termination and primer formation in mitochondria. J Biol Chem 281: 24647-24652.

Pohjoismaki JL, Goffart S. 2011. Of circles, forks and humanity: Topological organisation and replication of mammalian mitochondrial DNA. Bioessays 33: 290-299.

Pohjoismaki JL, Goffart S, Tyynismaa H, Willcox S, Ide T, Kang D, Suomalainen A, Karhunen PJ, Griffith JD, Holt IJ, et al. 2009. Human heart mitochondrial DNA is organized in complex catenated networks containing abundant four-way junctions and replication forks. J Biol Chem 284: 21446-21457.

Pohjoismaki JL, Holmes JB, Wood SR, Yang MY, Yasukawa T, Reyes A, Bailey LJ, Cluett TJ, Goffart S, Willcox S, et al. 2010. Mammalian mitochondrial DNA replication intermediates are essentially duplex but contain extensive tracts of RNA/DNA hybrid. J Mol Biol 397: 1144-1155.

Pomerantz RT, O’Donnell M. 2010. What happens when replication and transcription complexes collide? Cell Cycle 9: 2537-2543.

Reyes A, Yang MY, Bowmaker M, Holt IJ. 2005. Bidirectional replication initiates at sites throughout the mitochondrial genome of birds. J Biol Chem 280: 3242-3250. 
Samuels DC, Schon EA, Chinnery PF. 2004. Two direct repeats cause most human mtDNA deletions. Trends Genet 20: 393-398.

Santamaria D, de la Cueva G, Martinez-Robles ML, Krimer DB, Hernandez P, Schvartzman JB. 1998. DnaB helicase is unable to dissociate RNA-DNA hybrids. Its implication in the polar pausing of replication forks at ColE1 origins. J Biol Chem 273: 33386-33396.

Schatz G. 1963. The isolation of possible mitochondrial precursor structures from aerobically grown baker's yeast. Biochem Biophys Res Commun 12: 448-451.

Simsek D, Furda A, Gao Y, Artus J, Brunet E, Hadjantonakis AK, Van Houten B, Shuman S, McKinnon PJ, Jasin M 2011. Crucial role for DNA ligase III in mitochondria but not in Xrcc1-dependent repair. Nature 471: 245-248.

Spelbrink JN, Li FY, Tiranti V, Nikali K, Yuan QP, Tariq M, Wanrooij S, Garrido N, Comi G, Morandi L, et al. 2001. Human mitochondrial DNA deletions associated with mutations in the gene encoding Twinkle, a phage T7 gene 4-like protein localized in mitochondria. Nat Genet 28: $223-231$.

Topper JN, Bennett JL, Clayton DA. 1992. A role for RNAase MRP in mitochondrial RNA processing. Cell 70: 16-20.

Tyynismaa H, Sembongi H, Bokori-Brown M, Granycome C, Ashley N, Poulton J, Jalanko A, Spelbrink JN, Holt IJ, Suomalainen A. 2004. Twinkle helicase is essential for mtDNA maintenance and regulates mtDNA copy number. Hum Mol Genet 13: 3219-3227.
Van Goethem G, Dermaut B, Lofgren A, Martin JJ, Van Broeckhoven C. 2001. Mutation of POLG is associated with progressive external ophthalmoplegia characterized by mtDNA deletions. Nat Genet 28: 211-212.

Wanrooij S, Falkenberg M. 2010. The human mitochondrial replication fork in health and disease. Biochim Biophys Acta 1797: 1378-1388.

Wanrooij S, Goffart S, Pohjoismaki JL, Yasukawa T, Spelbrink JN. 2007. Expression of catalytic mutants of the mtDNA helicase Twinkle and polymerase POLG causes distinct replication stalling phenotypes. Nucleic Acids Res 35: 3238-3251.

Wolstenholme DR, Koike K, Cochran-Fouts P. 1974. Replication of mitochondrial DNA: replicative forms of molecules from rat tissues and evidence for discontinuous replication. Cold Spring Harb Symp Quant Biol 38: 267-280.

Yang MY, Bowmaker M, Reyes A, Vergani L, Angeli P, Gringeri E, Jacobs HT, Holt IJ. 2002. Biased incorporation of ribonucleotides on the mitochondrial L-strand accounts for apparent strand-asymmetric DNA replication. Cell 111: 495-505.

Yasukawa T, Yang MY, Jacobs HT, Holt IJ. 2005. A bidirectional origin of replication maps to the major noncoding region of human mitochondrial DNA. Mol Cell 18: 651-662.

Yasukawa T, Reyes A, Cluett TJ, Yang MY, Bowmaker M, Jacobs HT, Holt IJ. 2006. Replication of vertebrate mitochondrial DNA entails transient ribonucleotide incorporation throughout the lagging strand. EMBO J 25: 5358 5371. 


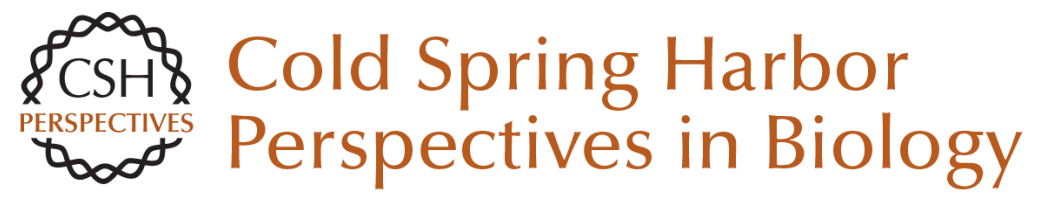

\section{Human Mitochondrial DNA Replication}

Ian J. Holt and Aurelio Reyes

Cold Spring Harb Perspect Biol 2012; doi: 10.1101/cshperspect.a012971 originally published online November 9, 2012

\section{Subject Collection DNA Replication}

Replication of Epstein-Barr Viral DNA Wolfgang Hammerschmidt and Bill Sugden

Replication Proteins and Human Disease Andrew P. Jackson, Ronald A. Laskey and Nicholas Coleman

Break-Induced DNA Replication Ranjith P. Anand, Susan T. Lovett and James E. Haber

Regulating DNA Replication in Eukarya Khalid Siddiqui, Kin Fan On and John F.X. Diffley

Archaeology of Eukaryotic DNA Replication Kira S. Makarova and Eugene V. Koonin

Translesion DNA Polymerases Myron F. Goodman and Roger Woodgate

Human Papillomavirus Infections: Warts or Cancer?

Louise T. Chow and Thomas R. Broker

Chromatin and DNA Replication

David M. MacAlpine and Geneviève Almouzni
Endoreplication

Norman Zielke, Bruce A. Edgar and Melvin L.

DePamphilis

Replication-Fork Dynamics

Karl E. Duderstadt, Rodrigo Reyes-Lamothe, Antoine M. van Oijen, et al.

Helicase Activation and Establishment of

Replication Forks at Chromosomal Origins of

Replication

Seiji Tanaka and Hiroyuki Araki

Poxvirus DNA Replication Bernard Moss

The Minichromosome Maintenance Replicative Helicase

Stephen D. Bell and Michael R. Botchan

DNA Replication Origins

Alan C. Leonard and Marcel Méchali

Principles and Concepts of DNA Replication in

Bacteria, Archaea, and Eukarya

Michael O'Donnell, Lance Langston and Bruce Stillman

DNA Replication Timing

Nicholas Rhind and David M. Gilbert

For additional articles in this collection, see http://cshperspectives.cshlp.org/cgi/collection/

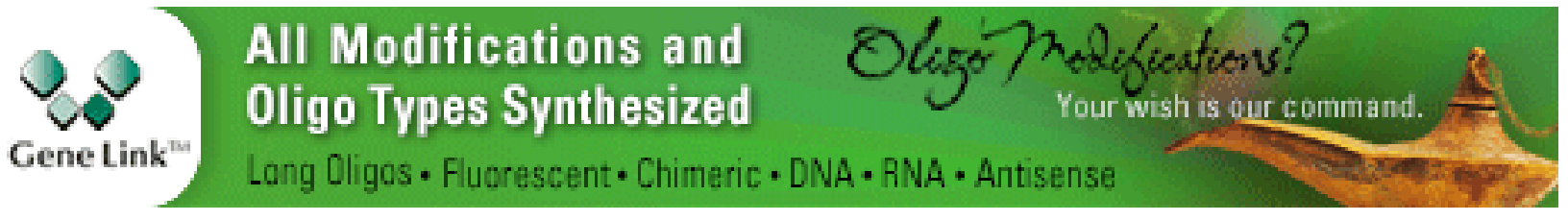

Copyright @ 2012 Cold Spring Harbor Laboratory Press; all rights reserved 